\title{
NP-External Arguments A Study of Argument Sharing in English
}

\author{
Adam Meyers, Ruth Reeves and Catherine Macleod \\ New York University \\ meyers/reevesr/macleod@cs . nyu .edu
}

\begin{abstract}
We explore some predicate-argument-structure phenomena in the context of the NomBank annotation project for English. Support verbs (They completed the acquisition), transparent nouns ( $\underline{\text { His }}$ first batch of questions), prepositions (At Mary's request, John left the room) and other lexical items can link arguments of a noun $N$ to positions outside of the NP headed by $N$. In these examples, They is an argument of acquisition, His is an argument of questions and John left the room is an argument of request. In most cases, these NP-external arguments are linked to a multiword expression (MWE) consisting of the noun predicate and (at least) one other item: a support verb, transparent noun, preposition, etc. This paper discusses properties of these constructions and how they interact. For example, in Disney made dozens of attempts to acquire Apple, Disney is an argument of acquire, due to linking properties of the support construction make + attempt and the quantificational noun dozens.
\end{abstract}

\section{Introduction}

Information extraction, question answering and other applications can benefit from a detailed analysis of noun predicate argument structure. For example, a high score in a management succession task (MUC, 1995) may entail that a system detect that Mary obtained a new job from either of the following sentences: (1) Mary $^{A}$ received the presidential appointment and (2) $\underline{I B M}^{S}$ gave ${ }^{\text {Mary }}{ }^{A}$ a promotion. ${ }^{1}$ A model of nominal predicate argument structure that goes beyond surface structure could be used to generalize existing patterns to cover these cases.

\footnotetext{
${ }^{1}$ We highlight featured nominal predicates in bold and underline their NP-external arguments. For exposition, we use superscripts to (approximately) distinguish subjects (S) from other arguments (A) and modifiers (M). Space limitations prevent us from breaking down argument structure as in NomBank.
}

Suppose that existing patterns associate the direct object of the verbs appoint and promote with the "incoming position slot". If the system is tuned to handle nominalizations in support verb constructions (Gross, 1981; Gross, 1982; Mel'čuk, 1988; Mel'čuk, 1996; Fontenelle, 1997; Macleod, 2002) such as receive the appointment and give a promotion, it would deduce that the surface positions of Mary in the above sentences map to the direct object positions of the verbs appoint and promote, and therefore also map to the "incoming position slot". In the enhanced model, a few patterns can be generalized for greater coverage.

Support constructions are an example of a more general phenomenon: arguments of a noun $N$ can occur outside of the NP headed by $N$, while the link between $N$ and these external arguments is predictable, typically because $N$ is part of a multiword expression (MWE). This type of MWE is interesting because it can take arguments that belong to other phrases, a more common property of non-MWE predicates, e.g., raising, equi and parenthetical verbs. This behavior should be viewed along with other behavior that was once viewed as atypical for MWEs. For example, in Mary pulled strings that nobody knew about to further her evil plan., it is clear that strings represents a decomposable part of the meaning (Nunberg et al., 1994). Discussion of such examples has led to an acceptance that the meaning of such idioms is decomposable. Similarly, other multiword expressions, like let alone in John doesn't like cheese, let alone pizza, have been shown to license whole constructions with complex syntax and scope/conjunction properties (Fillmore et al., 1988). It would seem that understanding the behavior we describe here is important to an understanding of what constitutes an MWE and how MWEs should be classified.

This paper explores the distribution of NPexternal arguments in the context of NomBank 
(Meyers et al., 2004b; Meyers et al., 2004a), a project with the goal of annotating all nominal argument structure in the Penn Treebank II corpus. The phenomena we cover include the following (among others):

- Support verb constructions like $\underline{I B M^{S}}$ gave Mary $^{A}$ a promotion; They ${ }^{S}$ took a walk; $\overline{\text { and } \underline{S h e}}^{A}$ had a visit.

- PP constructions exemplified by At Mary's request, John performed the operation ${ }^{A}$. and With ${\overline{J o ' s ~ h e l p, ~} \underline{F l o}^{A}}^{\text {discovered oil }}{ }^{A}$.

- Nominal constructions such as $\underline{h i s}^{A}$ share of the blame and $a$ topic $^{A}$ of discussion.

We also discuss how all these phenomena interact and sometimes form what we call support chains. For example, we can provide an explanation of how they is linked to the subject position of debate in the sentence They ${ }^{S}$ had lots of internal debate about this one. It turns out that the argument sharing aspects of the nominal phenomena we describe here is very similar to that of verbal phenomena which have been more throughly studied. Thus our example of a support chain is similar to subject equi (control) and subject raising examples like They seemed to want to debate, and our $\mathrm{PP}$ example with request is a lot like sentences with parenthetical verbs, e.g., John, Mary thought, could perform the operation really well. In both cases, the modifier of the sentence includes the sentence as an argument.

In this paper, we describe types and subtypes of the various constructions that allow noun arguments to occur outside the NP. In the process, issues of of "idiomaticity" arise. The phenomena discussed are typically licensed idiosyncratically by two or more words (an MWE). However, this is a tendency rather than a rule. For example, support verbs straddle the line between idioms and non-idioms (Sag et al., 2002). While keep tabs on and make sense really do seem to be idiomatic, some support verbs are quite productive, e.g., attempt can combine with many nouns that describe or imply actions, e.g., $\underline{H e}^{S}$ attempted + an attack/an experiment $/ \ldots$ Cases like give a standing ovation are decomposable - clearly something is being given, even though ovation is arguably the main predicate. Even cases like take a walk and make an attack should be viewed as MWEs, given that the light verbs take and make cooccur with only certain nouns - hence the ill- formedness of *make a walk and *take an attack. Thus the words and phrases that are the subject of this study range from non-MWEs to true MWEs and include many that are difficult to determine whether or not they should be considered MWEs. It is our view that phenomena are defined by their edges and not their middle and an understanding of the phenomenon discussed will help create better definitions and classifications of MWEs.

In the NomBank project, we annotate the argument structure of nouns, including these NP-external arguments. Creating specifications that are as unambiguous as possible would ensure that annotators record these phenomena consistently. This goal motivated us to make many of the definitions that we provide below.

\section{Support Verbs}

We define a support verb as a verb which takes at least two arguments $N P_{1}$ and $X P_{2}$ such that $X P_{2}$ is an argument of the head of $N P_{1}$. For example, in $\underline{J o h n}^{S}$ took a walk, a support verb (took) shares one of its arguments (John) with the head of its other argument (walk). A support verb can either be semantically empty, e.g., $\underline{J o h n}^{S}$ made a proposal to Mary or have content, e.g., Apple ${ }^{S}$ attempted a takeover of Sun Microsystems. ${ }^{2}$ The semantically empty support verbs, also called "light" verbs (Wierzbicka, 1982), are similar to subject to subject raising verbs like seem in John seemed to leave. In contrast, what we call the equi variety of support verbs are more like equi verbs (also called control verbs) like want in John wants to leave. In fact attempt can function either as an equi verb or as equi support verb, e.g., in both John attempted to attack and $\underline{J o h n}{ }^{S}$ attempted an attack, attempt shares its subject with attack. In all cases of support verbs, an argument is shared between a higher predicate $P_{1}$ and a lower predicate $P_{2}$, such that the phrase headed by $P_{2}$ is an argument of $P_{1}$. For light support verbs, $P_{1}$ carries modality, tense and other features, but is otherwise semantically empty - in particular the relation between the upper predicate and the shared argument is merely a "surface" relation, rather than a semantic one. In contrast, the equi-like support verbs assign a semantic role to the shared argument. Thus in the light support construction $\underline{J o h n}^{S}$ made an attack, John is just the

\footnotetext{
${ }^{2}$ Some researchers assume a narrow definition of support verbs that requires them to be semantically empty.
} 
ATTACKER, whereas in the equi support construction $\underline{J o h n}^{S}$ attempted an attack, John is both the ATTEMPTER and the ATTACKER.

The meaning and argument selection of a predicate nominal are often similar whether or not it occurs with a support verb. Nevertheless there are three important ways that support verbs and nouns interact. First of all, many support-verb/noun combinations qualify as MWEs. In general, only certain supportverb/noun combinations are possible, e.g., give $a$ kiss, but not ${ }^{*}$ make a kiss. Also, like idioms, some support-verb-plus-noun combinations allow aberrant syntax, e.g., make sense is fine without a determiner, whereas sense in other contexts requires one. Similarly, keep tabs on is only well-formed with the plural form of the noun tabs (compare $\underline{S h e}^{S}$ kept tabs on Mary with the ill-formed: *She kept a tab on $\overline{M a r y})$. These sort of limitations tend to occur in idioms. Some nouns do not occur outside the containing support construction, e.g., wreak havoc, suggesting that they are in fact idioms. Secondly, different support verbs have different argument sharing properties (Mel'čuk, 1988; Mel'čuk, 1996). For example, have a visit is different from pay a visit because the subject of have acts like the object of visit, but the subject of pay acts like the subject of visit. In $\underline{J o h n}{ }^{A}$ had a visit, someone visits John, whereas in $\underline{J o h n}^{S}$ paid a visit, John visits someone. Finally, arguments of a noun may have different interpretations depending on whether or not it occurs within a support construction. Some differences include the following:

- Negation and Modality - Support may change the implication as to whether or not an event actually takes place. A comparison of the following pairs of examples illustrates this point.

- Mary's destruction of the ring vs. Mary ${ }^{S}$ attempted the destruction of the ring

- Fred's nomination of Mary vs. Mary $^{A}$ refused Fred's nomination

- Degree of Agentivity - compare the shared argument's (Mary's) level of participation in the following phrases.

- Mary's attack on Fred

- Mary ${ }^{S}$ helped with the attack on Fred

- Mary ${ }^{S}$ planned an attack on Fred
In the first instance, Mary actually attacks; in the second, she helps attack; and if the attack even occurs in the third example, her role may be that of a general, not a soldier.

- Changes in Other Arguments - compare the extent of the destruction in the following two examples. In the first case, the destruction is total, but not in the second.

\section{- Mary's destruction of San Francisco \\ - Mary ${ }^{S}$ wrought destruction on San $\overline{\text { Francisco }}$}

Similar difference can be observed in raising and equi constructions. For example, John is the subject of attack in the following sentences: John attacked, John seemed to attack, John helped attack, John refused to attack. Nevertheless other aspects of meaning differ in each of these sentences, just as they do in the comparable support examples above.

Allowing for these various changes in meaning may make our definition of SUPPORT seem overly inclusive. However, we do not claim that support includes every case in which one can infer a connection between a noun phrase and one of its missing arguments. Rather, we require that the support relation be lexically based. This means that the support relation must either be licensed by the support verb, e.g., attempt or by the combination of the support verb, the noun and optionally, a preposition, e.g., keep tabs on. This rules out cases where a connection between a noun and one of its arguments can be made by other means. For example, in $\mathrm{He}$ did not want a rival to win the prize, one might deduce that $\mathrm{He}$ is an argument of rival. However, this connection is made by means of "bridging", a discourse process akin to coreference (Poesio and Vieira, 1998) - when not explicit, the argument of rival typically occurs nearby, just as the antecedent of he probably occurs nearby. Therefore rival is not part of a support structure in that sentence. Similarly, led is not a support verb in the sentence Market conditions led to the cancellation of the planned exchange. Here Market conditions is not an argument of cancellation - this would seem to violate a selection restriction since only sentient beings can cancel things. That sentence actually implies that Market conditions set a series of events into motion which ultimately caused the exchange to be canceled. 
Support constructions vary with respect to their degree of idiomaticity. Some support verb noun combinations seem really idiosyncratic, e.g., keep tabs on, make sense and wreak havoc. Others are quite productive, e.g., attempt and plan can occur with many different actions. Others are somewhere in the middle, e.g., take a walk and give a kiss. For some of the idiomatic and semi-idiomatic cases, it is not possible to cleanly make the equi/light distinction. For example, in $\underline{\text { John }}^{S}$ gave $\underline{M a r y}^{A}$ $a$ kiss and $\underline{J o h n}^{S}$ gave $\underline{\text { them }}^{A}$ a standing ovation, it is clear that John is the KISSER and the APPLAUDER, but it is unclear whether he is a GIVER or not. It depends on how literally you interpret the MWEs give a kiss and give a standing ovation. For annotation purposes, we do not draw this distinction as we are only interested in marking the argument structure of the nouns. Thus whether or not a shared argument is an "underlying" argument of the support verb as well is of little importance. ${ }^{3}$ Nevertheless, we note the distinction in order to provide annotators with a fuller inventory of support constructions. Knowing all the cases helps the annotators find the support constructions even though it is unnecessary to assign particular instances to one case or the other. On a similar note, our goal for annotation is to identify noun argument structure. It is not necessary for us to draw the line between productive support items and MWEs - we leave that to the user.

\section{Arguments across Copulas}

As shown in the following examples, prepositional and clausal arguments of nouns can usually occur in the environment:

[NP copula XP]

where XP is an argument of the head of NP. According to a literal interpretation of the above definition, these are instances of support. However, at best, they are trivial cases.

- The real battle is over who will control the market.

- This picture is about a middle-aged son who makes sure...

- The theory is that Seymour is the chief designer of the Cray-3...

\footnotetext{
${ }^{3}$ Similarly, the relation between the predicate "leave" and its argument John is the same in John tried to leave and John seemed to leave. It is only when one marks the higher predicates that a distinction is relevant.
}

Due to the generality of these constructions, it seems unlikely that these are really cases of support. Indeed it seems that cases of predication other than simple copular constructions can link nouns and arguments in the same way. The following includes examples of predication in clefting, extraposition, and small clauses. Therefore, we assume that these links are due to predication, not support.

- It's a mistake to put too much power in the hands of a single person. [CLEFT]

- The real battle will take place between center-stage players like Toshiba, Zenith and now Compaq. [EXTRAPOSITION]

- He considers it a mistake to buy anything from Fred. [SMALL CLAUSE]

\section{Partitives, Transparent Nouns, Crisscross Nouns, etc.}

There are a number of noun phrase constructions such that given the environment:

$\left[N P_{1} \ldots\left[\mathrm{PP}\left[N P_{2} N_{2}\right]\right]\right]$

an argument or adjunct of $N_{2}$ may precede the PP inside of $N P_{1}$. Below, we give the range of such cases that we have observed during our annotation effort. As we show later, the lower predicate $\mathrm{NP}_{2}$ can also be in prenominal positions. For simplicity we will stick with the PP cases in this section.

- $\underline{\text { Their }}$ S responsibility for hard decisions responsibility $=$ Support Noun, Their $=$ argument of decisions

- $\underline{H i s}^{S}$ attempt at the attack attempt $=$ Support Noun, His = argument of attack

- $\underline{H i s}^{S}$ first batch of questions batch = Transparent Noun, His = argument of questions

- $\underline{\mathrm{Her}}^{S}$ many avenues of research avenues $=$ Transparent Noun, $\mathrm{Her}=$ argument of research

- $\underline{\text { Its }}^{A}$ first stage of development stage $=$ a Part-Of noun, Its $=$ argument of development $t^{4}$

- Each company's share of liability share $=$ a Part-Of noun, each company $=$ argument of liability

\footnotetext{
${ }^{4}$ The verb develop participates in an alternation. Based on the transitive frame, its is the complement.
} 
The first two examples represent cases that are much like support verbs. In fact these nouns are nominalizations of the adjective responsible and the verb attempt which also have support/equi properties. In addition, so-called transparent nouns (batch, avenues) may act as bridges between a nominal predicate and its argument. Also, some part-whole relations allow this sort of argument transmission as well the possessor of a stage of development is the thing that is developing; if one has a share of liability, then one is (partially) liable.

In the following two examples, partitive constructions (quantifier $+o f$ ) interact with support verb constructions (make + decision and receive + award) to link an argument to a noun predicate. The quantifier plus of of the partitive constructions are sometimes analyzed as MWEs (complex quantifiers). ${ }^{5}$

- $\underline{S h e}^{S}$ made some of the decisions some = Partitive Quantifier, She = argument of decisions

- $\underline{\text { The film }}^{A}$ received many of the awards many $=$ Partitive Quantifier, The film $=$ argument of awards

Our final examples contain two types of nouns of an inside-out variety, the first of which we call CRISSCROSS nouns and the second we call ATTRIBUTE nouns:

- $\underline{\text { The victim }}^{A}$ of an assassination victim $=$ Crisscross Noun, The victim $=$ argument of assassination

- a subject ${ }^{A}$ of speculation $\overline{\text { subject }}=$ Crisscross Noun, a subject $=$ argument of speculation

- $\underline{\text { The bitterness }}^{M}$ of the battle Bitterness $=$ Attribute Noun, Bitterness $=$ manner modifier of battle

- The possibility $^{M}$ of an attack possibility = Attribute Noun, possibility = epistemic modifier of attack

A crisscross noun is an argument of its argument, i.e., the argument structures crisscross.

\footnotetext{
${ }^{5}$ The combinations $a$ lot of and lots of can be viewed alternatively as: (i) complex quantifiers, (ii) instances of the transparent noun lot or (iii) instances of partitives with the quantifiers $a$ lot and lots. It is almost impossible to choose between these alternatives. For our purposes, the second two choices have the same consequence - lot and lots can be part of support chains.
}

For example, victim is an argument of assassination in the victim ${ }^{A}$ of an assassination. ${ }^{6}$ Attribute nouns behave similarly. An attribute noun takes an argument $X$, such that the attribute represents some property of $X$, e.g., height, weight, etc. However, for some attribute nouns, there is an implication that $X$ possesses that attribute to some degree. When $X$ is an action, this implication may translate into an adverbial relation. For example, the phrase The possibility of an attack, can be used to quantify the chances that an attack will occur. In addition, it implies that the attack is possible (an epistemic modification relation).

\section{PP Constructions}

PPs consisting of particular prepositions plus nouns allow the noun to take some or all of the rest of the sentence as an argument, e.g., the preposition at in combination with request allows the rest of the sentence to act like the sentential argument of request in At Mary's request, John performed the operation ${ }^{A}$. The key factor to note here is that the combination of the preposition and the noun allow the argument of the noun to occur outside the noun phrase. Typically, only specific preposition plus noun MWEs license these constructions. As with other preposition plus noun MWEs, the determiner is often omitted, e.g., at issue, for enjoyment, without question.

We have observed at least three different varieties of PP constructions that allow NP-external arguments. First of all, there are preposition plus noun combinations that license extraposition structures. As shown in the following examples, these structures occur in the following three environments:

\section{i. Clause-or-NP copula PP}

ii. It copula PP Clause

iii. Subject-of-Infinitive copula PP to-infinitive The COMLEX Syntax (Macleod et al., 1998) class EXTRAP-P-NOUN-THAT-S identifies

\footnotetext{
${ }^{6}$ There seems to be an overlap between crisscross nouns and argument nominalizations of support verbs. The support construction receive a grant can be nominalized as grant recipients or recipients of grants. Here recipients is an argument nominalization of receive, the support verb, where an argument nominalizations refers to an argument (in this case the subject) of the corresponding verb. Grant is the crisscross noun argument of recipient in grant recipient and recipients of a grant, whereas (the incorporated argument of) recipient corresponds to the subject of receive a grant.
} 
members of this class of nouns along with the associated preposition. This PP construction seems to include a number of preposition + noun MWEs (of concern, at issue, at risk, to $X$ 's advantage, etc.) that license this set of complement structures. These PP units function similarly to adjectives that take extraposition complements, e.g., It is unfortunate that the party is over. Some examples follow: ${ }^{7}$

- What to do next ${ }^{A}$ is at issue [Clause-orNP copula PP]

- It was of concern that his fifth grade teacher released his records to the press ${ }^{A}$ [It copula PP Clause]

- Your experience ${ }^{A}$ may be of interest to the class [Subject-of-Infinitive copula PP toinfinitive]

The second variety of PPs are instances of subject oriented adverbials. Adverbs like willingly and accidentally in John accidentally spilled the beans and Mary left the room willingly; infinitival purpose clauses as in George lowered taxes to please his father and the PP expressions in the next set of examples have something in common: in addition to modifying the main clause (conveying manner or purpose), they also take the subject of the sentence as an argument. For example, in Mary left the room willingly, Mary is willing. In the examples below, we express the relation between the adverbial and the subject in terms of the argument taking properties of the prepositional object. In these examples, the PP is bracketed. Thus they is an argument of enjoyment; the jury is an argument of debate and she is an argument of memories. Of the PP constructions that we discuss here, this is the only type which we are aware of that arguably includes some non-MWEs. For example, prepositions like after can apply with a wide variety of action nouns (after the discussion/argument/battle) using normal syntax (the determiner is required for singular nouns).

- $\underline{T h e y}^{S}$ exercise [for enjoyment].

- [After much debate], the Jury ${ }^{S}$ focused on the facts.

- $\underline{S h e}^{S}$ would look back on this day [with fond memories].

\footnotetext{
${ }^{7}$ We assume that the that-clause (the second example) is part of the NP and therefore not an NP-external argument.
}

Finally, we have observed that some PPs are similar to a common variety of verbal parenthetical phrases, e.g., Mary, John assumed, was a biped or The origin of swiss cheese, Mary claims, is the island of Bomboolia. For verbs, these phrases are typically offset by commas and include a verb that takes a sentential complement. When the verbs occur in these parenthetical constructions, the sentential argument is filled by the main clause. ${ }^{8}$ Similarly, each of the examples below contain a PP (in brackets) containing a noun $N$, such that $N$ takes the rest of the sentence as an argument. We have observed that these PPs consist of: one of a small set of prepositions (at, with, without, and possibly a few others); and a noun that takes a sentential complement. The particular preposition/noun combinations allowed seem to be idiosyncratic - with the help of the FBI and at his request license this construction, but *at the help of the FBI and *with his request do not. Thus, these PPs seem to be limited to MWEs.

- The court hearing began in early October ${ }^{A}$ [at the request of Anthony Hazell]

- [With the help of the FBI], they taped their conversation ${ }^{A}$.

- [Without question], something intriguing is going on ${ }^{A}$.

In most cases, the main clause is an argument of the noun, much like the verbal cases mentioned above, e.g., The court hearing began in early October is the sentential argument of request above. However, the properties of the prepositional object determine the nature of its sentential complement and how these surface in the parenthetical construction. In the second example above, help takes two arguments: a participant and an action. These two arguments are realized respectively by the main clause subject and main clause predicate (VP). Finally, while not effecting argument structure some prepositions effectively negate the proposition. Thus, the main clause something intriguing is going on in the third example above is an argument of question; and the meaning of a logical proposition derived from this predicate must

\footnotetext{
${ }^{8}$ To be precise, the sentential argument is filled by the entire sentence, less the adverbial phrase itself. In other words, the sentential argument of assumed in the example above is Mary was a biped. Under the sloppier statement, it might appear that John assumed is part of the sentential argument. If so, semantic interpretation would enter an endless loop.
} 
include negation due to the preposition without, i.e., one might paraphrase the third sentence as One would not question that something intriguing is going on.

\section{Support Chains}

The phenomena described above can interact to form links in a chain. Consider the example: $\underline{I t}^{S}$ provides a source of marketing help. Provide is a support verb for help; source is a transparent noun; and help is an equi support noun. This entails that: (i) It is an argument of help due to the interaction of the support construction and the transparent noun source; and (ii) It is an argument of marketing (It helped market something) due to the equi support noun help. Further examples follow:

- The Big Board ${ }^{S}$ is considering a variety of actions.

- They $^{A}$ took advantage of dozens of opportunities.

- $\underline{I t}^{S}$ provides a source of marketing help.

- $\underline{I t}^{S}$ makes only a handful of decisions.

- $\underline{\text { The students }}^{S}$ took part in the demonstrations.

- $\underline{S a a b}^{A}$ is looking for a partner ${ }^{S}$ for financial cooperation.

We have observed a number of properties of support chains including: (1) There can be at most one verb in the support chain. This must be the first item in the chain, the one that governs the shared argument in the surface structure of the sentence; (2) Partitive quantifiers cannot be the first item in a support chain. These contrast with similar transparent nouns which can be, e.g., $\underline{H i s}^{A}$ source of marketing help; (3) The shared argument can be a logical argument of more than one link in the support chain, where $X$ is a "logical argument" of $Y$ means something like $Y$ assigns a theta role to $X$ in many theories. For example, consider They $^{A}$ took advantage of dozens of opportunities. They is an argument of both advantage and opportunities because in interpreting this sentence it is important to note that they achieved an advantage and that they are taking opportunities. In contrast, there is no similar relation between they and dozens and one's interpretation of the predicate took, depends on whether one views "take advantage" as idiomatic (as described above) - assuming that advantage is the main predicate and take is a light verb, then one would claim that take bears no logical relation with They; (4) Support chains can be idiomatic, e.g., to take part $i n$; (5) When a crisscross noun is part of a support chain, the crisscross noun itself must be an argument of the lower predicate. For example, partner is a crisscross noun in the example above $\left(\underline{S a a b}^{A}\right.$ is looking for a partner ${ }^{S}$ for financial cooperation.) Here $S a \overline{a b}$ and the hypothetical partner are both arguments of the lower predicate cooperation. This is possible because crisscross nouns often take an additional argument which is shared by the lower predicate. For example, in Mary's $S$ assassination victim, Mary is an argument of both assassination and victim.

\section{Miscellaneous Interactions}

This section describes miscellaneous examples of other interactions among the phenomena described above.

\subsection{PP constructions with transparent nouns}

The PP constructions discussed in section 5 can interact with transparent nouns. For example, consider: After hours of debate, they ${ }^{S}$ focused on the facts. This sentence begins with a subject-oriented PP modifier. The transparent noun hours serves as a link for the predicate debate. Thus debate, rather than hours actually takes they as an argument (the subject).

\subsection{Multiple Support Verbs}

Relative clauses and reduced relative clauses may contain support verbs. This opens up the possibility that the same noun may co-occur with more than one support verb. In all the cases of this that we have observed in our corpus, the support verbs fill in different argument slots of the same event, although in theory they could introduce different events - A hypothetical instance would be something like, $\underline{H e}^{A}$ suffered the same humiliation that $\underline{I}^{A}$ had experienced many years ago. Here, the noun humiliation refers to two distinct events.

The following sentence represents the more typical case of multiple support verbs:

Fred $^{S}$ charged $\underline{\text { Enron }}^{A}$ with squandering its assets $^{A}$ in a complaint filed in federal court ${ }^{A}$. $\overline{\text { In our analysis, the sense of complaint that }}$ refers to a legal action takes four arguments: the claimant, the reason for the complaint, the 
adjudicator (court of law), and the defendant. The sentence above includes all four arguments. There are two support verbs. The matrix verb charged links complaint to three of the arguments: Fred = the claimant, Enron $=$ the defendant and squandering its assets $=$ the reason. The remaining adjudicator argument (federal court) is linked to complaint by the support verb filed which heads a reduced relative clause.

\section{Conclusion}

We have presented data which we believe is crucial to an understanding of the argument structure of nouns and certain MWEs that include argument-taking nouns. Specifically, we have shown that there are certain lexical triggers for linking arguments of nouns across various phrase boundaries. By using these lexical triggers, we believe that many NLP applications (Information Extraction, Question Answering, etc.) will be able to detect noun arguments that would be lost otherwise, thus improving results.

Our account is based on work in theoretical syntax and NLP on support verbs and transparent nouns. To these phenomena, we add additional phenomena, particularly crisscross nouns and our PP parenthetical constructions. To our knowledge, ours is the first account that discusses how these phenomena interact to form support chains. Argument sharing phenomena in nouns turn out to be very similar to previously studied argument sharing phenomena in verbs (raising, equi, parentheticals, etc.).

A final feature of this work is the place of the nominal argument sharing phenomena we describe in work on MWEs. Many support verb + noun combinations either qualify as bona fide idioms or straddle the line between idioms and non-idioms. Our hope is that a better understanding of the argument taking properties of these structures will help with the argument structure of idioms and MWEs more generally.

\section{References}

C. J. Fillmore, P. Kay, and M. C. O'Connor. 1988. Regularity and Idiomaticity in Grammatical Constructions: The Case of Let Alone. Language, 64:501-538.

T. Fontenelle. 1997. Turning a bilingual dictionary into a lexical-semantic database. Lexicographica Series Maior 79. Max Niemeyer Verlag, Tübingen.

M. Gross. 1981. Les bases empiriques de la notion de prédicat sémantique. In A. Guillet and C. Lecl'ere, editors, Formes Syntaxiques et Prédicat Sémantiques, volume 63 of Langages, pages 7-52. Larousse, Paris.

M. Gross. 1982. Simple Sentences: Discussion of Fred W. Householder's Paper "Analysis, Synthesis and Improvisation". In Text Processing. Text Analysis and Generation. Text Typology and Attribution. Proceedings of Nobel Symposium 51.

C. Macleod, R. Grishman, and A. Meyers. 1998. COMLEX Syntax. Computers and the $\mathrm{Hu}$ manities, 31:459-481.

C. Macleod. 2002. Lexical annotation for multiword entries containing nominalizations. In Proceedings of the 3rd International Conference on Language Resources and Evaluation (LREC-2002), Las Palmas, Spain.

I. A. Mel'čuk. 1988. Dependency Syntax: Theory and Practice. State University Press of New York, Albany.

I. A. Mel'čuk. 1996. Lexical Functions: A Tool for the Description of Lexical Relations in a Lexicon. In Lexical Functions in Lexicography and Natural Language Processing. John Benjamins Publishing Company, Amsterdam.

A. Meyers, R. Reeves, C. Macleod, R. Szekely, V. Zielinska, B. Young, and R. Grishman. 2004a. Annotating Noun Argument Structure for NomBank. In Proceedings of LREC2004, Lisbon, Portugal.

A. Meyers, R. Reeves, C. Macleod, R. Szekely, V. Zielinska, B. Young, and R. Grishman. 2004b. The NomBank Project: An Interim Report. In NAACL/HLT 2004 Workshop Frontiers in Corpus Annotation, Boston.

MUC-6. 1995. Proceedings of the Sixth Message Understanding Conference. Morgan Kaufman. (MUC-6).

G. Nunberg, I. A. Sag, and T. Wasow. 1994. Idioms. Language, 70:491-538.

M. Poesio and R. Vieira. 1998. A Corpusbased Investigation of Definite Description Use. Computational Linguistics, 24:183-216.

I. A. Sag, T. Baldwin, F. Bond, A. A. Copestake, and D. Flickinger. 2002. Multiword expressions: A pain in the neck for NLP. In CICLing, pages 1-15.

A. Wierzbicka. 1982. Why can you have a drink when you can't *have an eat? Language, 58:753-99. 